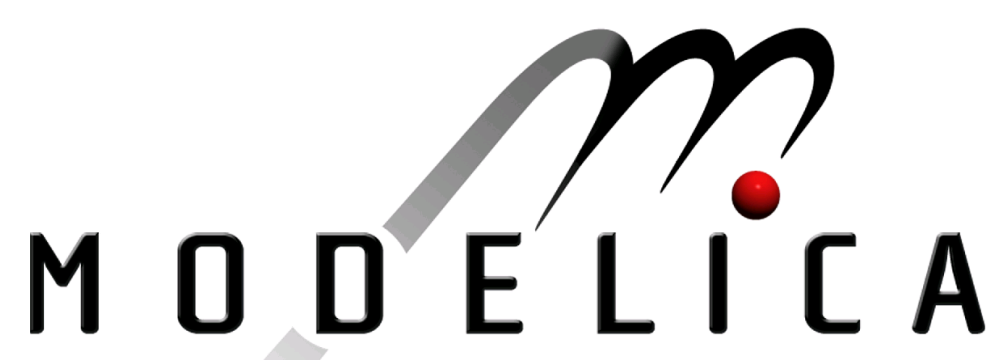

Proceedings of the 4th International Modelica Conference, Hamburg, March 7-8, 2005, Gerhard Schmitz (editor)

L.J. Yebra, M. Berenguel, S. Dormido, M. Romero CIEMAT-PSA; Universidad de Almeria; UNED, Spain Modelling and Simulation of Central Receiver Solar Thermal Power Plants pp. $413-421$

Paper presented at the 4th International Modelica Conference, March 7-8, 2005, Hamburg University of Technology, Hamburg-Harburg, Germany, organized by The Modelica Association and the Department of Thermodynamics, Hamburg University of Technology

All papers of this conference can be downloaded from http://www.Modelica.org/events/Conference2005/

Program Committee

- Prof. Gerhard Schmitz, Hamburg University of Technology, Germany (Program chair).

- Prof. Bernhard Bachmann, University of Applied Sciences Bielefeld, Germany.

- Dr. Francesco Casella, Politecnico di Milano, Italy.

- Dr. Hilding Elmqvist, Dynasim AB, Sweden.

- Prof. Peter Fritzson, University of Linkping, Sweden

- Prof. Martin Otter, DLR, Germany

- Dr. Michael Tiller, Ford Motor Company, USA

- Dr. Hubertus Tummescheit, Scynamics HB, Sweden

Local Organization: Gerhard Schmitz, Katrin Prölß, Wilson Casas, Henning Knigge, Jens Vasel, Stefan Wischhusen, TuTech Innovation GmbH 


\title{
Modelling and Simulation of Central Receiver Solar Thermal Power Plants
}

\author{
L.J. Yebra ${ }^{\dagger} * * \quad$ M. Berenguel ${ }^{*} \quad$ S. Dormido M. Romero $^{\dagger}$
}

${ }^{\dagger}$ CIEMAT-PSA. Ctra. de Senés s/n. Tabernas.

E04200 Almería. Spain. E-mail:\{luis.yebra,manuel.romero\}@psa.es

*Universidad de Almería. Dpto. de Lenguajes y Computación. Ctra. de Sacramento s/n. La Cañada. E04120 Almería. Spain. E-mail: beren@ual.es

¥U.N.E.D. Escuela Técnica Superior de Ingeniería Informática. Dpto. Informática y Automática. C/ Juan del Rosal, 16. E28040 Madrid. Spain. E-mail: sdormido@dia.uned.es

\begin{abstract}
The implementation of advanced control systems to optimize the overall performance of Central Receiver Solar Thermal Power Plants is nowadays a priority research line in CIEMAT-PSA. The development of dynamic models for use in simulation and control of this kind of power plants is presented in this article, focused on the CESA-I solar plant. The developed model is based in the thermohydraulic modelling framework ThermoFluid, and the main components of the system are presented as well as the respective modelling assumptions. A typical operating cycle is simulated and the results are shown and commented.
\end{abstract}

\section{Introduction}

This paper presents the current status of the research performed within the framework of modelling and simulation of Central Receiver Solar Thermal Power Plants (CRSTPP). The work is mainly oriented to the development of dynamic models of solar energy plants to be used in the design of automatic control systems aimed at optimizing global performance. The models presented in this article are focused on the solar part, excluding typical components of power plants like turbines or generators.

The system used as test-bed plant is the CESA-I facility, a CRSTPP belonging to the CIEMAT (Centro de Investigaciones Energéticas, Medioambientales y

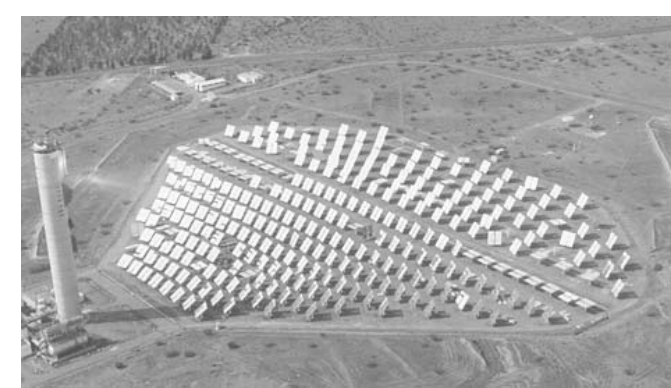

Figure 1: CESA-I solar plant at PSA

Tecnológicas - Research Centre for Energy, Environment and Technology), public organism owned by the Spanish Ministry of Science and Education. This solar plant is located at the Plataforma Solar de Almería (PSA), South-East Spain. A join project between CIEMAT-PSA, the University of Almería (UAL), the National University of Distance Education (UNED) and the University of Seville (US) is being carried out in order to develop models and control systems to automatically control these kind of plants. This test-bed plant can be seen in figure 1 .

The model presented in this paper will be used in the design of hybrid model predictive control and intelligent control schemes to optimize plant performance, even under start-up and shutdown situations and in the presence of highly variable load disturbances due to the daily cycle of solar radiation and passing clouds. 


\section{Central Receiver Solar Thermal Power Plants}

In this section an overview of the basic components and operating procedures for a CRSTPP is introduced. Figure 2 shows an explicative diagram of a general CRSTPP.

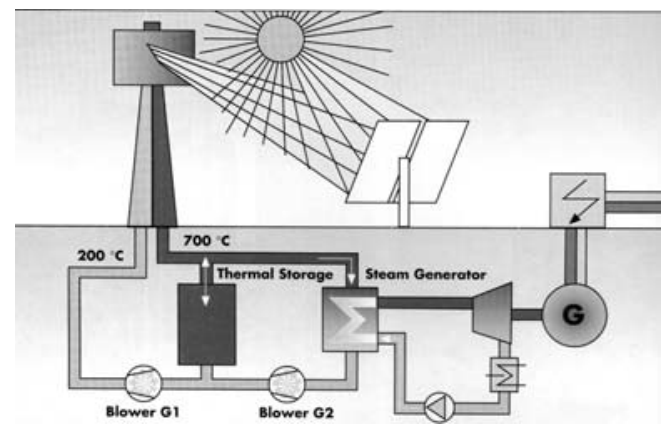

Figure 2: Schematic diagram of a CRSTPP

The operation of this kind of plants is based in the concentration of incoming solar energy using a heliostat field that reflects the incident solar radiation onto a (typically volumetric) receiver (theoretically onto an optical point in the 3-D space). As the sun position changes during the day, each heliostat of the field (composed of 300 heliostat in the case of the CESA-I plant) has to change its position in real time according to the selected aiming point on the receiver, as different aiming points can be selected in order to achieve a uniform temperature distribution on the receiver [7]. The receiver is located at the top of the tower $(84 \mathrm{~m}$ height in CESA-I) and acts as an energy exchanger, receiving solar energy and transferring it to a thermohydraulic circuit with air medium, see figure 2 . The system is also composed by an energy storage tank, an air/water-steam heat exchanger (evaporator), blowers and valves. The combined action of the blowers let to feed either the storage tank or the heat exchanger with hot air. The evaporator is formed by the primary circuit and a secondary one with subcooled inlet water and with superheated steam outlet. A measurement of the overall concentrated input radiation, a controlled water pump and an outlet controlled valve define the main boundary conditions for the system. The final objective of the model is to predict the transient behaviour of the thermodynamics variables associated to the thermohydraulic output power of the evaporator (mainly temperature, pressure, and specific enthalpy), when the external disturbances (concentrated solar radiation, ambient temperature, and wind speed and direction) and controllable inputs (both at the solar field level: number of operating heliostats and aiming point strategy followed, and at the tower level: mass flow rate demanded by the blowers, inlet water flow and outlet pressure valve position) change.

\section{Modelling of CRSTPP}

In this paper we will concentrate in the thermohydraulic part of the system, skipping the heliostat field and assuming a known input radiation power in the receiver as a consequence of the radiation reflected in the heliostat mirrors and the aiming-point strategy followed [7]. This assumption introduces an error in the estimated irradiation due to the difficulties that exist in getting accurate high concentrated radiation measurements.

Due to the fact that the main phenomena are related to thermofluids, the Modelica language has been used to develop these models including the ThermoFluid library ([12],[6]) as a framework over which create own libraries and final component models. The authors think that this library is an important reference in the framework of object oriented modelling of thermofluid systems with Modelica, and its existence makes a nonsense to develop libraries in the same level of modelling of thermohydralic systems.

The work analyzes each of the components of the thermohydraulic circuits of air and water-steam and explain the modelling assumptions, trying to justify each one as they are oriented to get, by means of the symbolic manipulations that Dymola tool performs, a not high index DAE system for the complete model, in which the number of nonlinear algebraic loops is minimized. For this purpose, all the components are classified, following the modelling methodology derived from the Finite Volume Method (FVM) [10], in Control Volumes (CV in ThermoFluid nomenclature) and Flow Models (FM in ThermoFluid nomenclature). In some cases information about the future control system architecture to be implemented is introduced in the modelling phase. An example of components that are modelled using this kind of information are the blowers in the air circuit, in which a cascade control will help avoid the multivariable nonlinear dependence of the constitutive equations and let consider them like quasi ideal flow rate generators. Due to the existence of components whose internal implementation may vary depending on the modelling hypotheses, the polymorphism and the Modelica language constructs replaceable/redeclare have been specially used in some of them, for example in the evaporator. 
Figure 3 shows the developed Modelica model of the system.

The following components are shown: blowers, storage tank, solar receiver, evaporator, control valve, sensors, pipes and connections. All of them are directly instantiated and parameterized, or inherited from ThermoFluid classes. It can be seen how the air circuit is composed of a solar receiver, tubes, tank, and evaporator; and the water steam circuit with a water pump, a control valve, tubes, and reservoirs with boundary conditions. In both circuits the mass transfer connections have been drawn with solid and thick lines, while colors are used to describe hot fluid (red), cold fluid (blue), and hot steam (grey). Input-Output causal connectors appear to access variables of interest for control purposes like:

Output variables. Boolean indicator for satu$\mathrm{rated} / \mathrm{superheated} \mathrm{vapor,} \mathrm{several} \mathrm{pressures} \mathrm{and}$ temperatures in both media circuits, stored energy in the tank, mass flow rate in the tank, mean temperature in the tank, etc.

Input variables. Actuators setpoints: blowers 1 and 2 , water pump; and boundary conditions: pressure and temperatures of water inlet, estimated inlet concentrated radiation, ambient temperature and pressure, wind velocity.

\subsection{ThermoFluid usage}

The thermohydraulic interface is formed by connectors from ThermoFluid for single component media and steady-state momentum balance statement. Due to the fact that the dynamics of momentum balance are of no interest for control purposes in the time scales studied at present, the stationary formulation version of ThermoFluid has been used in all the thermohydraulic classes utilized.

The modelling methodology adopted from the beginning for the design of the classes was: if there exists any class in ThermoFluid that implements the physical phenomenon to model, use it with the corresponding parameters; if not, design the classes using inheritance from the high level partial classes from ThermoFluid; in other cases then use proper ThermoFluid interfaces and base classes and develop the component class with the lacking behavior expressed in differential and algebraic equations from first principles. In the next subsections the most important components models will be detailed and the modelling hypotheses will be explained and justified.

\subsection{Blowers and Pumps}

In this kind of active FM [12], the authors decided to make a simplifying assumption based on the gained experience in control of Parabolic Trough Fields with thermal oil as medium, case of Acurex field of CIEMAT-PSA [4], [5], and water-steam as medium in DISS facility [16], [13]. This assumption is that the water pump and blowers are controlled in a cascade scheme [3] with a local control loop whose dynamics is much faster than the rest of the thermohydraulic system. This assumption has been experimentally validated in blowers and water pump, and helps simplifying these components models until the possibility of state them as quasi-ideal mass flow rate generators. This approximation lets avoiding the consuming time work of fitting the nonlinear multivariate curves of the pumps and blowers. So, the algebraic equation for these components is $\dot{m}=\dot{m}_{r e f}$, where $\dot{m}_{r e f}$ is the setpoint of the local pump/blower control loop and is assigned in a connector to the model, as can be seen in figure 3.

\subsection{Solar Receiver}

This component receives energy from the heliostat field, that concentrates solar radiation in different aiming points to avoid large spatial temperature gradients that could damage the component. This aiming point strategy is a research line at present, [7], and is implemented in the heliostat field real time control system. The total energy flow reflected by the heliostats and concentrated in the receiver is nowadays estimated, because the practical difficulties in the measurement of this variable. Therefore, a mean solar concentrated input power is used for modelling purposes. This assumption introduces uncertainty in the model, that makes not to expect from the adjusted and validated model a precision beyond that of the inlet power. Nevertheless, it is expected that this uncertainty will decrease in the near future with the development and implementation of new sensors and by re-calibrating the model with new operating data.

Figure 4 shows the Modelica model composed, in a major number, of ThermoFluid components. This is the model of the system in which the strongest simplifications have been made, due to the internal design of the system. From a system level point of view, the receivers are composed of an arrangement of solid ceramic cups that receive inlet radiation. Due to hydraulic depression caused by external pumping elements (blowers 1 and 2), an air mass flow rate from 


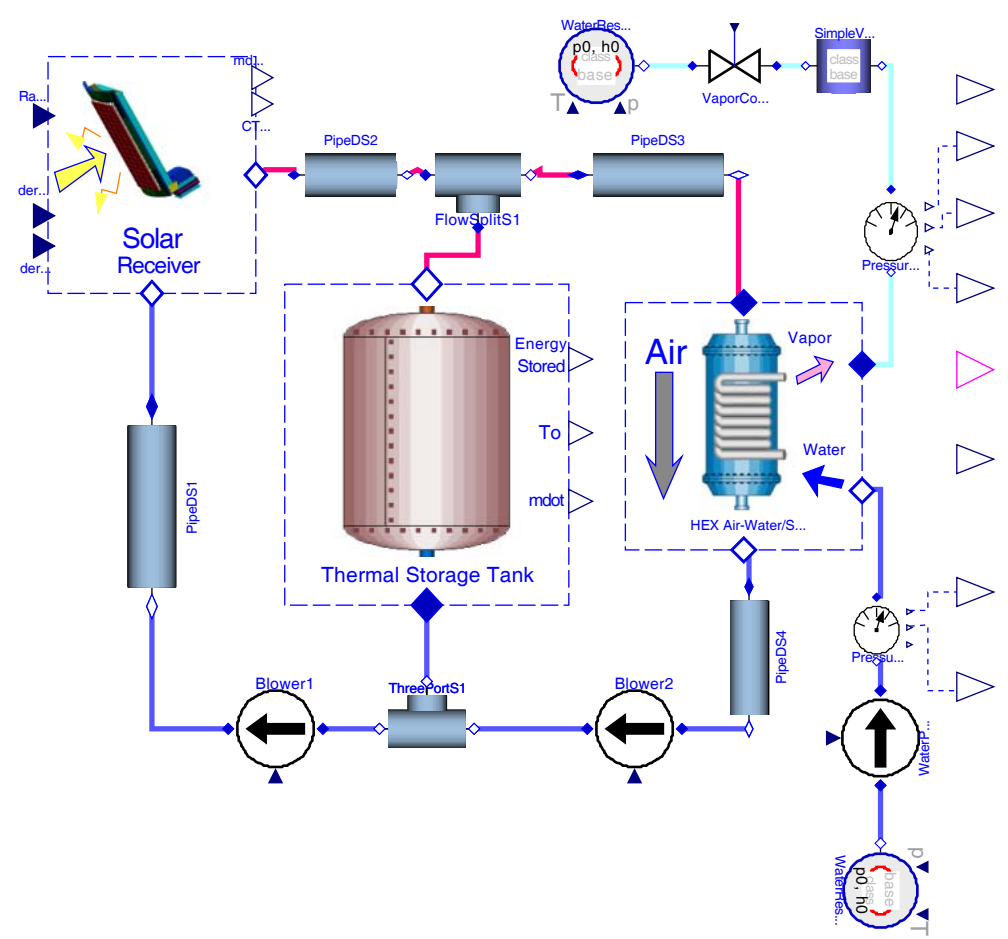

Figure 3: Modelica model of CESA-I CRSTPP

the environment is established and heated when passing through the ceramic cups. The cups are modelled by wall classes, the air mass and energy conservation by concentrated parameters CV's and the medium model for air from medium models. The external boundary conditions are modelled by reservoir componentes representing external pressure and temperature. The conduction-convection and radiation energy flow from receiver to atmosphere is modelled by heat transfer classes with the extensions of include expressions for empirical correlations obtained by experimentation. The convection heat transfer between the $\mathrm{CV}$ and the cup array is based in empirical correlations too. The radiation and convection are modelled using the Stefan-Boltzmann Law and Newton Cooling Law [9], in which the radiation conductance in the former case, and the heat transfer coefficient, in the latest, are the variables to identify by means of adjusted correlations. The three input connectors represent: concentrated solar radiation in the receiver (radin), derivative of atmospheric pressure and derivative of ambient temperature, respectively. The former is the estimated concentrated solar radiation, and the second and third are used as sources of uncertainties to the model. The derivative of the pressure lets simulate experimented effects that wind gusts could cause in the pressure and temperature close to the receiver. The explicit modelling of disturbances caused by wind are important

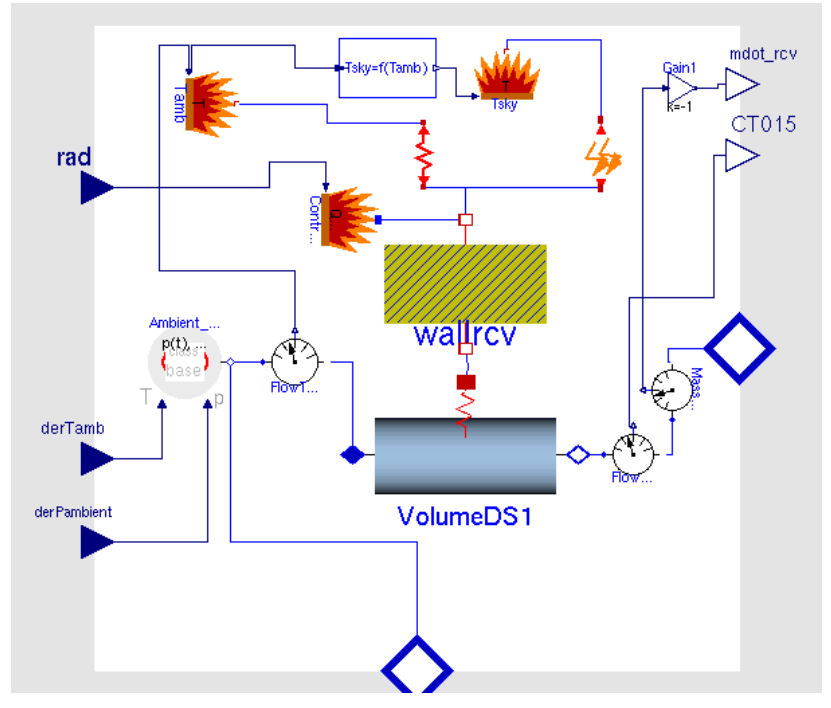

Figure 4: Modelica model of CESA-I receiver

for control purposes of the temperature of the air leaving the receiver to the circuit.

\subsection{Storage Tank}

The aim of the storage tank is to accumulate energy to let the plant operate when irradiation decreases during a time interval (limited by the tank capacity and layout). The tank can be defined by three states, depending on the mass flow rates of the blowers, see figure 


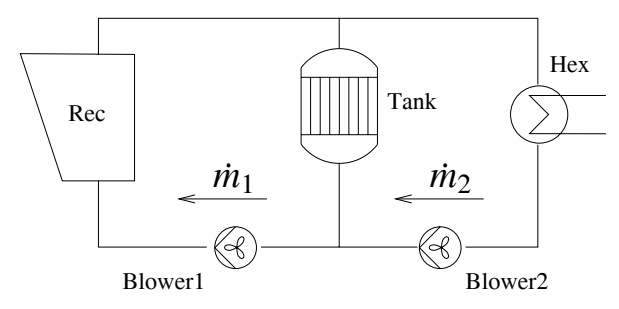

Figure 5: Schematic diagram of the air circuit

5 , and assuming enough incoming power from the receiver and negligible energy losses to the environment from the tank:

Loading: $\dot{m}_{1}>\dot{m}_{2}$. The energy stored in the tank is increasing.

Unloading: $\dot{m}_{1}<\dot{m}_{2}$. The energy stored in the tank is decreasing.

Standby: $\dot{m}_{1} \approx \dot{m}_{2}$. The stored energy does not vary noteworthily.

The tank is filled with a solid media that is exposed to thermal contact with air that flows through the tank. Between the air and the solid media there is an energy exchange by convection phenomena that is described by the Newton Cooling Law and the energy conserved in the air flow and the solid media. Due to the spatial distribution of the tank, it is modelled as a parameter distributed system in the direction of the major dimension of the tank. This results in a system of partial differential equations (PDE) formed by:

- Differential formulation of mass and energy conservation through the air volume in contact with the media, in the domain defined along the major spatial dimension of the tank. See [11].

- Differential formulation of energy conservation of the solid media, in the domain defined along the major spatial dimension of the tank. See [11].

To solve the PDE system stated, it is necessary to spatially discretize the equations. ThermoFluid provides partial classes [12] in which the discretization with the Finite Volume Method (FVM) ([10]) is applied. One of these classes is ThermoFluid.PartialComponents...Volume2PortDS_pT,

which implements this mass, energy and static momentum conservation equations in a volume spatially discretized in $n$ subvolumes. For the solid media, there exists final use classes that implements energy conservation in distributed solids, ThermoFluid.Components.HeatFlow.Walls.

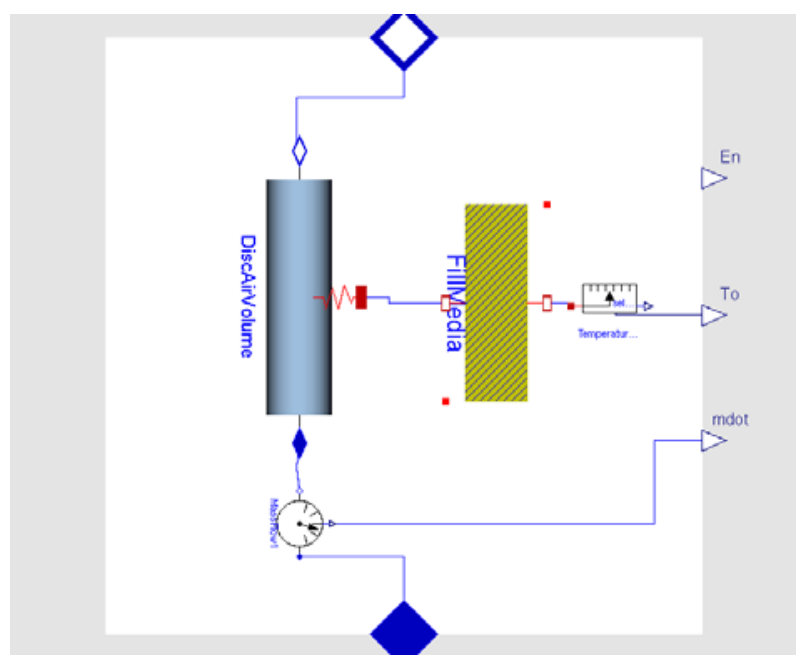

Figure 6: Model of the Storage Tank

To close the system of equations it is mandatory to introduce the heat transfer coefficient between the air flow and the solid media. This coefficient depends of heat transfer correlations using adimensional fluid numbers (Reynold, Prandtl, Pecklet,...), geometry of the contact surface and thermodynamic and transport properties of the fluid (air in this case). Some of the correlation parameters strongly depend on the experimentation and parameter adjusting phase of the modelling work. See [11].

The tank model, designed using ThermoFluid components, is shown in figure 6 , where the discretized air volume component (DiscAirVolume), the solid media component (FillMedia) and the component modelling the heat transfer coefficient ${ }^{1}$ can be observed.

The additional variables that are get out through output connectors for control purposes are:

- En : total stored energy in the solid media with respect to a reference level.

- $T_{0}:$ spatially averaged temperature of the solid media.

- $\dot{m}$ : air mass flow rate through the tank.

\subsection{Evaporator}

The evaporator in CESA tower is a counterflow airwater/steam hex, in which the water/steam flow is he-

\footnotetext{
${ }^{1}$ In this version of the model, the heat transfer coefficient is supposed to be constant. Including a dependence of this coefficient using correlations does not pose any additional difficulty, but redesigning a discretized volume for air, polymorphic with the first one, with one variable and one equation for the heat transfer correlation, e.g., Dittus-Boelter [11].
} 
licoidal configured through the air flow. For modelling effects, this components has been considered as a counterflow hex composed of one pipe with air media, one pipe with water-steam media and a wall letting thermal interaction. The simple arrangement can be seen in figure 7 .

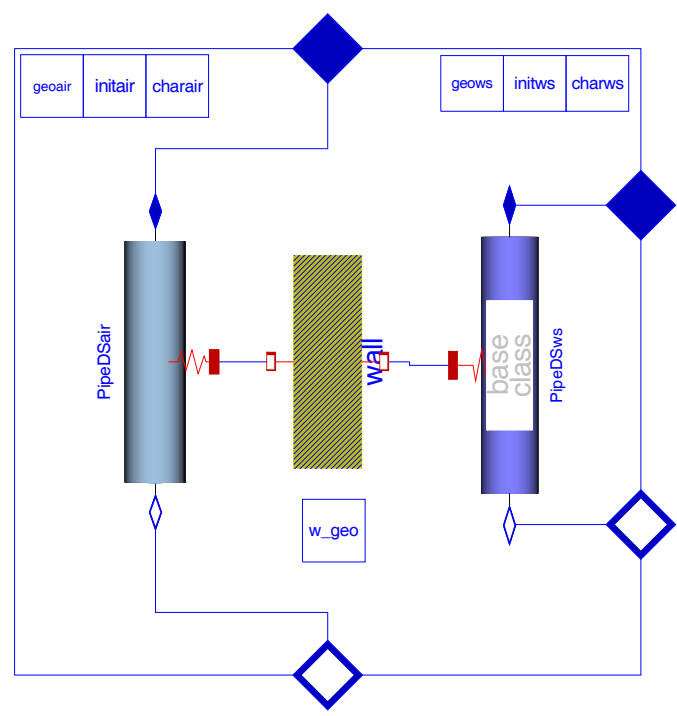

Figure 7: Discretized model of the Evaporator

The length of the water/steam pipe is $1440 \mathrm{~m}$. and under normal operating conditions the inlet water is in subcooled region and the outlet vapor is superheated; thus the dynamical conditions will vary along the pipe depending on the thermodynamic and transport properties of the water/steam. The configuration shown in figure 7 is fully discretized in $n$ CV's in which mass,energy, and momentum balances are applied? The number of CV's, $n$, is a trade off between accuracy and computing cost, so the final choice is the minimum $n$ that models dominant dynamics for control purposes. Nowadays we are working with values in the interval $[10,15]$. The wall and the air pipe are discretized with the same discretization level.

In the development of experimental correlations classes for the heat transfer coefficients sliding models and chattering have appeared with some frequency around the phase changes of water/steam CV's. This phenomena are manifested with more frequency when CV's pass from subcooled (region 1 in IAPWS-IF97 standard for water/steam properties, [15]) to saturated (region 4 in IAPWS-IF97), due to the discontinuities present in the heat transfer coefficients in the limit boundary between water and walls. To avoid those cases in which chattering causes troubles to the sim-

\footnotetext{
${ }^{2}$ Momentum conservation is stated in staggered CV's with respect to those ones which state mass and energy conservation; [10], [14] and [12].
}

ulation, another polymorphic evaporator model has been developed, in which the subcooled and saturated regions of water/steam pipe are replaced by an equivalent Moving Boundary Model (MBM) [8]. Figure 8 shows this mixed discretized and MBM model, where the MBM component has been designed with ThermoFluid interfaces to be connected with the rest of components.

Although the mixed model lowers the likelihood of finding chattering in the integration process, it is theoretically less accurate, and experimentally it is harder to find consistent DAE initial conditions and the validity range of the model is more limited than that of the fully discretized one.

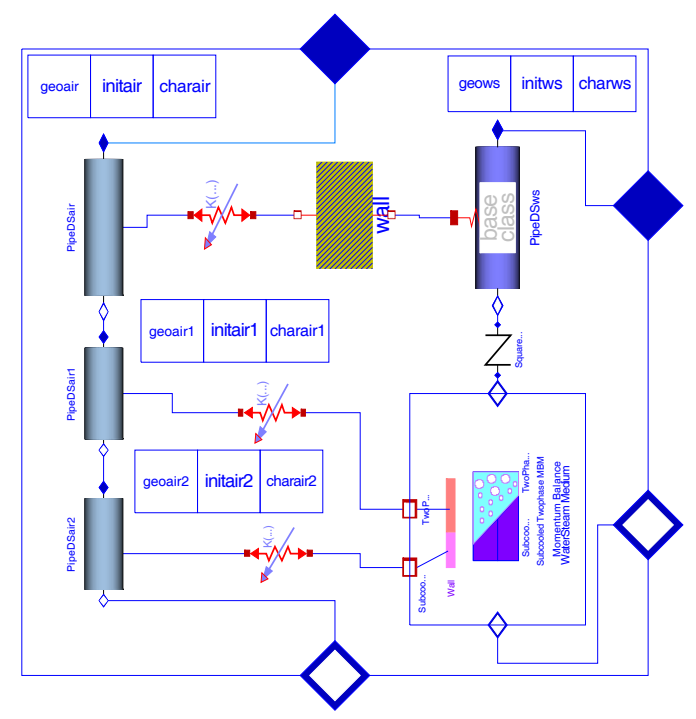

Figure 8: Mixed Moving Boundary and Discretized model of the Evaporator

With the help of replaceable/redeclare constructs and the choices annotations ([2], [1]), the switching between fully discretized and mixed MBM-discretized models in instantiation time easies the modelling work.

\subsection{Simplified model}

Some components of the compound model have been introduced to maintain the structure and topology in the model similar to the real system, following the object oriented approach. Some of them, like pipes, actually introduce additional differential equations in the model that could be eliminated due to the fact that the parameters of the real plant make their time constants and delays too low when compared to the rest of components. Eliminating the pipes implies assuming negligible energy losses and fast dynamics in the mass conservation due to the low real volume, which is rea- 
sonable. The model could be simplified from figure 3 to the one shown in figure 9.

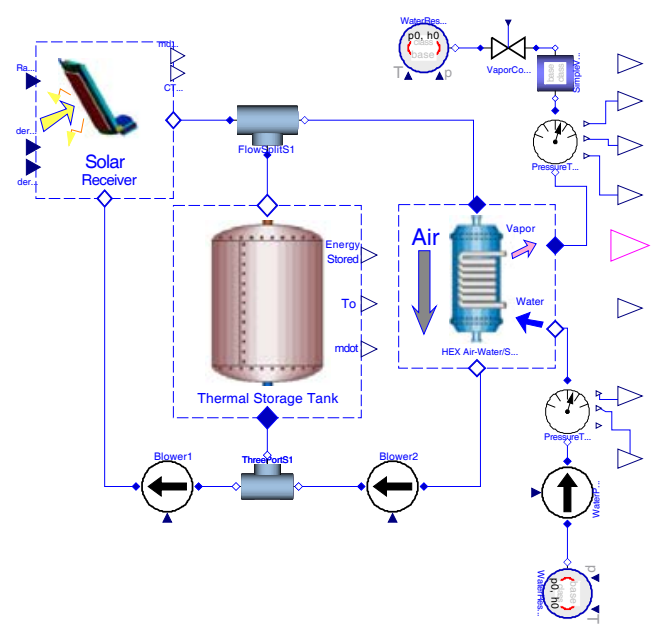

Figure 9: Simplified model of figure 3

\section{Simulation}

This section shows representative simulation results using the simplified plant model of figure 9 , when the system executes the cycle detailed below, that represents a typical operation case:

1. Initial state: the storage tank is unloaded, the air circuit is at ambient conditions and the water circuit is in subcooled region.

2. At time $=0 s$, an input power of $10 \mathrm{MW}$ is reflected from the heliostat field in the receiver and the storage tank begins to accumulate energy. No power is delivered to the evaporator.

3. At time $=2000 s$, all the energy from the receiver is delivered to evaporator and the storage tank energy level remains unchanged.

4. At time $=15000 \mathrm{~s}$, the power from the heliostat field is zero (due to a passing cloud, for example) and all the energy delivered to the evaporator comes from the storage tank.

The results of this simulation are shown in figure 10. The first graph presents the input power radiation (Power_Rad) from the heliostat field. The second the mass flow rates in blowers 1 (mdotBlower1) and 2 (mdot_Blower2), delivering energy to the storage tank or evaporator in each time interval. The third graph shows the load/unload cycle of the storage tank; it can be seen how the dominating time constants for load/unload are different and that the accumulated energy can be enough to maintain the outlet superheated steam during some time. The fourth graph presents the outlet evaporator water/steam temperature; this augments when the blower 2 works until the solar radiation exists, then begins to fall at low rate while stored energy remains in the tank. When the energy coming from the tank is not enough to maintain the superheated steam, it get saturated and then subcooled. The last graph presents the IAPWS-IF97 regions crossed by outlet water/steam travel during this simulation; it can be seen that initially the water is subcooled (region 1 ), then enters in saturation (region 4), then enters in superheated (region 2); when the solar radiation disappears at time $=15000 \mathrm{~s}$, the storage tank maintains the superheating state some time until the steam enters in saturation again (region 4), and finally come back to subcooled water (region 1) as during the beginning of the process. At present, no real plant data are allowable for model calibration and validation purposes. Conducted tests are planned to validate each one of the components of the model.

\section{Concluding remarks and Ongoing work}

This article shows the development of a model of a CRSTPP using the methodology of object oriented development of thermofluid systems. The major part of the components are based in the ThermoFluid framework for thermohydraulic modelling. The CRSTPP components and main operation principles have been described. For the main components, the modelling hypotheses and the composition Modelica diagrams developed with the Dymola tool have been presented. References to the underlying physical phenomena have been made in these composition diagrams, without entering in detail of quantitatively describing them through differential and algebraic equations; instead, the basic bibliography and the ThermoFluid classes that implements them have been referenced. Finally, a simplified model showing a typical operation cycle with a real perturbation introduced by clouds has been simulated.

The ongoing works to develop consists in the adjusting of the main block models parameters based in the experimental results of the real plants, by means of working separately with each shown component with its proper boundary conditions. In this work, the validation of empirical correlations for heat transfer and 

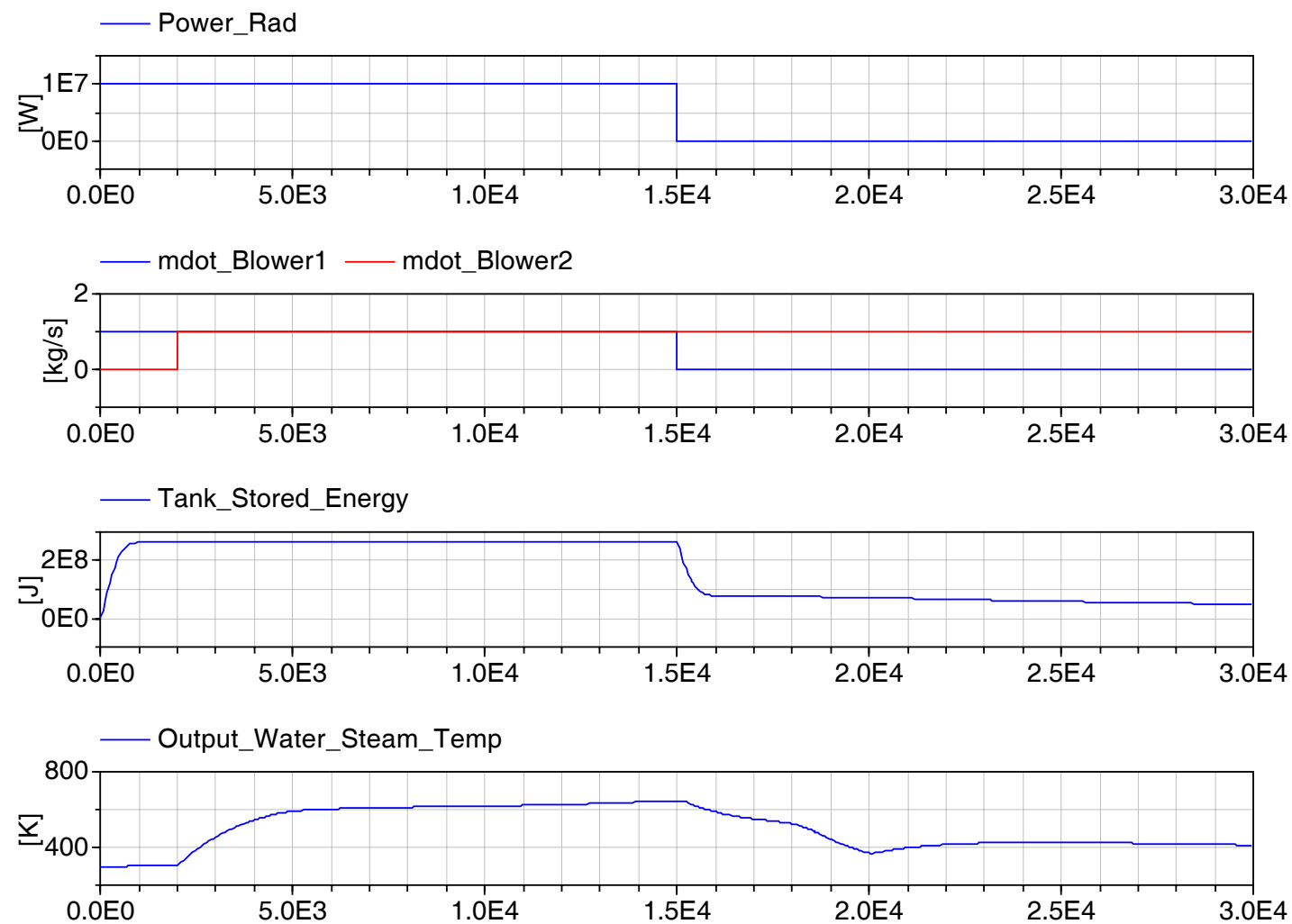

—IAPWS_IF97_region_Output_Water_Steam

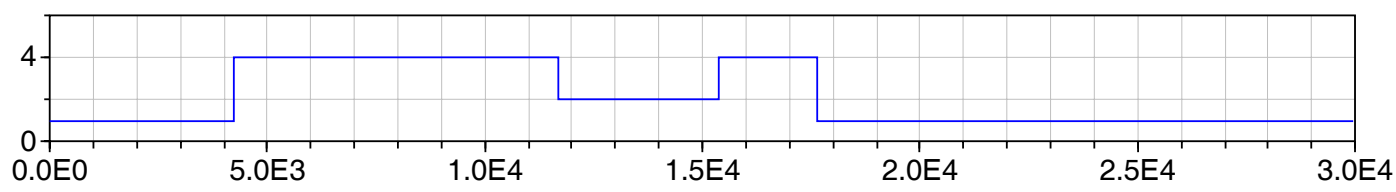

Figure 10: Simulation results. 
pressure loss will be an important issue.

The final aim is to develop control and automatic operation systems that help operating in the most autonomous way this kind of plants, in the presence of large disturbances. Automatic start-up and shutdowns of the plants is one of the main objectives in this direction.

\section{Acknowledgements}

This work has been financed by CICYT-FEDER funds (projects DPI2001-2380-C02-02, DPI200204375-C03, DPI2004-07444-C04-04 and DPI20011012) and by the Consejería de Innovación, Ciencia y Empresa de la Junta de Andalucía. This work has been also performed within the scope of the specific collaboration agreement between the Plataforma Solar de Almería and the Automatic Control, Electronics and Robotics (TEP-197) research group of the Universidad de Almería titled "Development of control systems and tools for thermosolar plants".

The authors want to acknowledge to J.D. Alvarez for his help with some of the bitmaps of some Modelica models developed. Of course, thanks to Dr. Jonas Eborn, Dr. Hubertus Tummescheit and Lund Technology Institute for the development and make public the ThermoFluid library.

\section{References}

[1] Dynasim AB. Dymola Users Manual. Version 5.3a. Dynasim AB, Research Park Ideon. SE22370 Lund. Sweden.

[2] Modelica Association. Modelica. a unified object-oriented language for physical systems modeling. language specification. version 2.1. Technical report, Modelica Association, January 2004.

[3] K.J. Åström and B. Wittenmark. ComputerControlled Systems. Prentice Hall, 1997.

[4] M. Berenguel. Contributions to the control of distributed solar collectors (in Spanish). $\mathrm{PhD}$ thesis, Escuela Superior de Ingenieros Industriales de Sevilla. Spain, March 1996.

[5] E.F. Camacho, M. Berenguel, and F.R. Rubio. Advanced Control of Solar Plants. Springer, 1997.

[6] J. Eborn. On Model Libraries for Thermohydraulic Applications. PhD thesis, Department of Automatic Control, Lund Institute of Technology, Sweden, March 2001.

[7] F.J. Garcia-Martín, Berenguel M., Valverde A., and E.F. Camacho. Heuristic knowledge-based heliostat field control for the optimization of the temperature distribution in a volumetric receiver. Solar Energy, 66:355-369, 1999.

[8] J.M. Jensen and H. Tummescheit. Moving boundary models for dynamic simulations of two-phase flows. In Martin Otter, editor, Proceedings of the $2 r d$ International Modelica Conference, pages 235-244, Oberpfaffenhofen, Germany, March 2002.

[9] Kreith, editor. The CRC Handbook of Thermal Engineering. CRC Press, 2000.

[10] S.V. Patankar. Numerical Heat Transfer and Fluid Flow. Series in Computational and Physical Processes in Mechanics and Thermal Sciences. Taylor \& Francis, 1980.

[11] W.M. Rohsenow, J.P. Hartnett, and Y.I. Cho. Handbook of Heat Transfer. McGraw-Hill, 1998.

[12] H. Tummescheit. Design and Implementation of Object-Oriented Model Libraries using Modelica. PhD thesis, Department of Automatic Control, Lund Institute of Technology, Sweden, August 2002.

[13] L. Valenzuela, E. Zarza, M. Berenguel, and E.F. Camacho. Direct steam generation in solar boilers. IEEE Control System Magazine, 24(2):1529, 2004.

[14] H.K. Versteeg and W. Malalasekera. An Introduction to Computational Fluid Dynamics. Addison Wesley Longman Limited, 1995.

[15] W. Wagner and A. Kruse. Properties of water and steam. Springer-Verlag, Berlin, 1998.

[16] E. Zarza. The Direct Steam Generation with Parabolic Collectors. The DISS project (in Spanish). $\mathrm{PhD}$ thesis, Escuela Superior de Ingenieros Industriales de Sevilla, November 2003. 